\title{
Effect of Virgin Coconut Oil Supplementation on Obese Rats' Anthropometrical Parameters and Gut Bacteroidetes and Firmicutes Change Ratio
}

\author{
Amadeus Driando Ahnan ${ }^{1^{*}}$, Widya Agustinah ${ }^{1 \pi}$
}

\begin{abstract}
Previous clinical trials using virgin coconut oil (VCO) showed a potential anti-obesity effect, including for human, by reducing the waist circumference. However, there was not discovered any research that examined its anti-obesity effect in relation with gut microbiota parameters as one of current approaches of obesity parameters, for example Firmicutes and Bacteroidetes. This research was conducted to examine the effect of VCO supplementation on the change of Firmicutes and Bacteroidetes in comparison to the anthropometrical parameters. The experiment was done using 14 days obese-induced female Sprague-Dawley rats supplemented with $0.01 \% \mathrm{w} / \mathrm{w}$ and $0.02 \% \mathrm{w} / \mathrm{w}$ VCO for 28 days. The anthropometric data collection consisted of body weight (BW), body mass index (BMI), abdominal circumference (AC), and body length (BL). Ratio changes in -fold increase between Day 28 and Day 0 of Bacteroidetes and Firmicutes were retrieved by using relative quantification normalized against unit mass calculation, using the cycle threshold values from real-time polymerase chain reaction (RT-PCR) method with SYBR Green fluorescence and standard curve derived from tenfold 5 points dilution series of recombinant plasmid for efficiency calculation. VCO supplementation stimulated significant increase on anthropometric parameters on BW and AC ( $<<0.05)$, while insignificant changes were shown on Bacteroidetes and Firmicutes ratio with tendency of increasing and decreasing respectfully, with no significance. The result indicated that VCO supplementation in the given doses promoted pro-obesity effect on key anthropometric parameters, while showed potential anti-obesity effect on the gut microbiota parameter. Further researches were needed especially in determining the effective dose to promote the anti-obesity effect of VCO and revealing any possible cellular and molecular mechanisms.
\end{abstract}

Keywords: Virgin Coconut Oil, RT-PCR, Firmicutes, Bacteroidetes, Obesity

\footnotetext{
${ }^{1}$ Faculty of Biotechnology, Atma Jaya Catholic University of Indonesia, Jakarta, Indonesia.

"Corresponding author

E-mail: driando.ahnan@gmail.com (ADA)

Mailing address: Jl. Garnisum Dalam 8, Sudirman Tower Condominium C-11-C, Semanggi, Jakarta 12930, Indonesia.
}

${ }^{\text {II }}$ Supervisor 


\section{Introduction}

Coconut (Cocos nucifera) was known as one of the most popular natural sources that are abundant of medium chain fatty acids (MCFA), a group of fatty acids that has been suspected for anti-obesity effects. The effects were evidenced by the supplementation of virgin coconut oil (VCO), which was responsible for adult male human respondents' circumference significant reduction (Liau et al., 2011). A similar lipidlowering effect was found towards the rats that were supplemented by VCO by their lower bodyweight, tissue cholesterol, and tissue triacylglycerol (Srivastava et al., 2013). On the other hand, there had been emerging novel approaches on obesity diagnosis, prevention, and treatment. One of the most novel points of view to analyze obesity is through metagenomics, which corresponds to the study of a collection of genetic material from a mixed community of organisms and often be useful to represent unculturable organisms (National Human Genome Research Institute, 2015). The metagenomic analysis was important for anticipating obesity because the composition of some mostly unculturable groups bacteria, i.e. Firmicutes and Bacteroidetes phyla, showed a correlation with obesity by acting as the core gut microbiome (Turnbaugh et al., 2006).

\section{Obesity-related Gut Microbiota}

Gut microbiota is a complex community consisted of bacteria, eukaryotes, viruses and Archeae, which inhabits gastrointestinal tract (GIT) of organisms, including mammals like humans and rats. Bacteroides, Clostridium, Eubacterium, Ruminnococcus, and Bifidobacterium were some examples of bacteria groups that were commonly found in the intestines and digestive tract (Bäckhed et al., 2005). Approximately 50 different phyla in the intestine had been identified with Bacteroidetes, Firmicutes, and Actinobacteria as the three dominant phyla (Mariat et al., 2009). The composition of gut microbiota could affect its host metabolism and physiological function. Certain autoimmune diseases, cancer, obesity, autism, and other physiological factors had been linked to gut microbiota composition and its alteration (Turnbaugh et al., 2009; TlaskalováHogenová et al., 2011). In human and rat studies, obese individuals contained higher number of Firmicutes and lower number of Bacteroidetes in their gut compared to lean individuals (Ley et al., 2006). The research exemplified how gut microbiota caused metabolic change due to its contribution to energy harvesting ability. One of the mechanisms was by producing short chain fatty acids (SCFA) as the product of fermenting undigested fiber, which the SCFA then can be used as an energy source by the liver, muscle, and adipose tissue (Schwiertz et al., 2010).

Firmicutes was described as a bacteria phylum that consisted of mostly Gram-positive bacteria with low mol\% $\mathrm{G}+\mathrm{C}$ of their DNA, generally less than $50 \%$. The phylum Firmicutes consisted of more than 26 families and 223 genera, which all possessed rigid cell wall due to the content of muramic acid for all and teichoic acid for some. The phenotype of the members was diverse. The cells might be spherical, straight, curved, with helical rods or filaments, with or without flagella, and with or without heat-resistant endospores. They were aerobes, facultative or strict anaerobes. Some of the Firmicutes were thermophiles and/or halophiles. Most of them were chemo-organotrophs, and few were acidophiles or alkaliphiles in which most grew at neutral pH (Vos et al., 2011).

The phylum Bacteroidetes consisted of phenotypically diverse group of Gram-negative rods bacteria that do not form endospores and mostly anaerobic. The phylum contained four classes, Bacteroidia, Cytophagia, Flavobacteria, and Sphingobacteria. In addition, Rhodothermus, Salinibacter, and Thermonema were deep groups that could not be readily assigned to any of the four classes. Some of the members of Bacteroidetes phylum are motile by flagella or gliding (Krieg et al., 2011).

The opposite role of the two phyla was shown by that high-fat diet increased the number of Firmicutes and decreased the number of Bacteroidetes, while high-carbohydrate but lowfat diet caused the opposite. Through KEGG metabolic pathway relative abundance clustering, it was revealed that Bacteroidetes' 
genome was enriched with carbohydrate metabolism pathways, while Firmicutes' genome was enriched with transport systems pathways. Genes those were found abundant in Bacteroidetes were glycoside hydrolases, carbohydrate- binding modules, glycosyltransferases, polysaccharide lyases and carbohydrate esterases; while those were found abundant in Firmicutes were methionine metabolism genes, ABC transporters, transporters phosphotransferase system (PTS), selenoamino acid metabolism, and also valine, leucine and isoleucine biosynthesis (Turnbaugh et al., 2009).

\section{Virgin Coconut Oil and Obesity}

VCO is the oil obtained from fresh and mature kernel of coconut (Cocos nucifera) by mechanical or natural means that does not alter the nature properties of the oil. VCO-making should not undergo chemical refining, bleaching, or deodorizing. VCO can be consumed in its natural state without further processing (Asia Pacific Coconut Community, 2015). VCO can also be used for cooking and topical applications such as for hair blackening and wound healing (Nevin and Rajamohan, 2006). Examples of processes that can be used to produce VCO are by heating or centrifuging coconut milk, pressing coconut kernel, and letting the air-borne Acetobacter spp. to inoculate the coconut milk. Such processes broke the emulsion of the coconut milk, making the oil separated from other components like carbohydrates and proteins (Srivastava et al., 2013).

VCO is relatively popular due to its high MCFA contents (53-75\% of total fatty acids), especially lauric acid (45-46\% of total fatty acids) (Asia Pacific Coconut Community, 2015). MCFA is consisted of $8-12$ carbon chains (C8C12) thus be metabolized differently than long chain fatty acid (LCFA). LCFA was absorbed by the intestinal cells then transported by the lymph system and chylomicrons to subcutaneous tissue, which led to subcutaneous fat tissue formation. Differently, MCFA was not transported to the lymph, otherwise straight to the liver to be readily used as an energy source through $\beta$ oxidation, which possible increased the energy expenditure rate of the body (Guturu and Duchini, 2012). In human lipid digestion system, fatty acids itself might be derived from diet in the form of free fatty acids or as the results of triglyceride digestion by lipase (Zimmermann et al., 2004).

\section{Recent Obesity Examination Development}

Obesity is an abnormality of excess fat accumulation that may impair individual health. Obesity is determined by a calculation of body mass index (BMI). For human, BMI is retrieved by the calculation of weight in kilograms divided by the square of height in meters $\left(\mathrm{kg} / \mathrm{m}^{2}\right)$, which is classified as obese if the BMI is greater or equal to $30\left(\mathrm{~kg} / \mathrm{m}^{2}\right)$ (World Health Organization, 2015). For rats, similar calculation differed as the weight in grams divided by the square of height in centimeters $\left(\mathrm{g} / \mathrm{cm}^{2}\right)$. While there was no particular standard for obese classification, a research classified rats as obese if the BMI is greater or equal to $0.68\left(\mathrm{~g} / \mathrm{cm}^{2}\right)$ (Novelli et al., 2007).

Human obesity cases had increased to more than double since 1980. In 2014, over 600 million adults out of more than 1.9 billion overweighed adults were obese. Obesity was dominated for about $13 \%$ out of $39 \%$ of overweighed adults in the world in 2014. Most of the world's populations lived in the countries where overweight and obesity killed more people than the underweight. Even in children, 42 million children under the age of 5 were overweight or obese in 2013 (World Health Organization, 2015).

Obesity was generally caused by an energy imbalance between calories consumed and calories expended, for example, an increased intake of energy-dense food that was high in fat and an increase of sedentary nature. However, keeping the energy consumption lower than the energy expenditure could prevent obesity. This can be achieved through limiting energy intake, increasing physical activity, and also increasing the proportion of fiber-rich foods (World Health Organization, 2015). Not only caused by lifestyle, other factors that affected obesity were genetic and epigenetic. There were 54 loci found to have associations with obesity, mainly 
affected the phenotypes of BMI, waist circumference, and waist-hip ratio. The epigenetic factors related to obesity were mostly DNA methylation and histone modification (Herrera et al., 2011).

Recently, obesity has been connected with gut microbiota. Obese individuals had more Firmicutes and less Bacteroidetes number compared to lean individuals (Turnbaugh et al., 2009). Beside that, it was also observed that children who became overweight by age 7 had lower levels of Bifidobacteria and higher levels of Staphylococcus aureus as infants compared with those with healthy weight (Kalliomäki et al., 2008). A test using gut microbiota transplantation on mice also showed that mice colonized with microbiota originated from obese donors exhibited a significantly greater percentage increase in body fat over two weeks compared to mice colonized with microbiota from lean donors (Ley et al., 2006).

\section{RT-PCR Analysis}

RT-PCR is an advancement of polymerase chain reaction system that can detect, amplify, and quantify DNA sample. RT-PCR continuously collects fluorescent signal from one or more polymerase chain reactions over a range of temperature cycles. The conversion of the fluorescent signals from each reaction can be converted into a numerical value for each sample as quantification data. The fluorescence intensity is proportional to copy number of DNA that is formed in the PCR process. RT-PCR uses cycle threshold $(\mathrm{Ct})$ as a measurement unit to determine the intensity of the fluorescent in proportion with the samples amount, for example the copy number of DNA. Ct is the cycle where enough intensity of fluorescent can be detected by the optical sensor. Given that, the higher the $\mathrm{Ct}$ number is, the lower the DNA sample concentration is (Dorak, 2007). Each RT-PCR instrument applies particular specification. One of the highly used fluorescent is SYBR green, which has the wavelength of $300 \mathrm{~nm}$ (Lekanne Deprez et al., 2002).

By its ability to retrieve copy number of DNA sample by interpreting its copy number, RT-PCR could be used to quantify the number of bacteria cells in the gut by assuming one copy DNA represents one cell of bacteria. This metagenomic technique was considered helpful to quantify unculturable bacteria like Firmicutes and Bacteroidetes for their anaerobes nature (Guo et al., 2008).

\section{Previous Examinations and Research Objective}

Various researches had been conducted to examine the effect of dietary supplementations on the change of gut microbiota, especially using sources of prebiotic because of its survivability to reach the intestine tract undigested. Prebiotic feeding decreased Firmicutes number, increased Bacteroidetes number, and might modulate certain gut hormones (Everard et al., 2011). The fermentation of prebiotics by gut microbiota produced short chain fatty acids (SCFA) that was used as the source of energy and also as hormone stimulator. Butyrate, as a type of SCFA, was the main source of energy for gut microbiota. It could also bind to the receptors of enteroendocrine cells to stimulate gut hormones, which modulated gut transit time, recovery of SCFAs from diet, and fat accumulation in mice fat pads (Ley, 2010).

However, it had not been found yet any publications that studied how VCO would affect the change in gut microbiota. Related researches about VCO and obesity were Liau et al. that showed VCO supplementation reduced the waist circumference significantly on adult males, and Srivastava et al. that showed VCO supplementation on rats lowered the BW (Liau et al., 2011; Srivastava et al., 2013).

The objective of this research was to evaluate the effect of VCO supplementation on Firmicutes and Bacteroidetes composition of obese female Sprague Dawley (SD) rats. This research hypothesize that VCO supplementation would cause the increase in Bacteroidetes and decrease in Firmicutes, as the opposite effect compared to the relation to obesity reported (Turnbaugh et al., 2006, 2009). The possibility of gut microbiota modulation as a factor of VCO's anti-obesity effect was determined in this study. 


\section{Materials and methods}

The molecular analysis of this research was conducted at DNA Technology Laboratory, Faculty of Biotechnology, Atma Jaya Catholic University of Indonesia, Jakarta, Indonesia. The rat treatment was conducted at Pusat Studi Satwa Primata, Bogor Agricultural University primate research center, Bogor, West Java, Indonesia. The research was started on July 2014 and finished on January 2015.

\section{Ethics Statement}

This study was approved by the ethic commission of Pusat Studi Satwa Primata, Bogor Agricultural University primate research center, Bogor, West Java, Indonesia.

\section{VCO Preparation}

The VCO used in this study was made through modified kitchen method (Srivastava et al., 2013). Coconut milk derived from grated old coconut at Pasar Bogor, West Java, Indonesia, was heated on low-heat until the emulsion was broken down and the oil could be separated and collected manually.

\section{Animal Study}

Five to six weeks-old female SD rats were used in this research. A total of 27 SD rats were divided into 3 treatment groups of 9 individuals each i.e. control group $(0.01 \% \mathrm{w} / \mathrm{w}$ distilled water), group $1(0.01 \% \mathrm{w} / \mathrm{w} \mathrm{VCO})$, and group 2 $(0.02 \% \mathrm{w} / \mathrm{w} \mathrm{VCO})$, all by oral gavage. The use of distilled water as control is aligned to the hypothesis of this study itself, which is that VCO as an exceptional fat would give anti-obesity effect even though that means adding more calorie by the supplementation. Similar designs were also shown in the previous researches that reported anti-obesity effects of VCO. Liau et al. added VCO (30 mL/day for 1 month) to basal adult human diet instead of using reference oil, so did Srivastava et al. who added VCO $(+10 \%$ or $+15 \%$ for 15 days) to rats' basal diet instead of using reference oil (Liau et al., 2011; Srivastava et al., 2013).

The sample size was reduced to 7 per group due to extreme outliers of two samples found in Bacteroidetes parameter thus led to the elimination in each group to equalize (S1 and S2 Tables). Those treatments of supplementation were performed for 28 days (Day 1 to Day 28). Earlier, the rats were supplemented with $20 \%$ $\mathrm{w} / \mathrm{v}$ sucrose for 14 days as pre-treatment to induce obesity (Day -14 to Day 0 ). The VCO dose was determined by converting the effective dose of $30 \mathrm{~mL}$ for human (Liau et al., 2011) using the Foods and Drugs Administration (FDA) human-rat equivalent dose calculation (Food and Drug Administration, 2005) resulting $\pm 3.25 \mathrm{~mL} / \mathrm{g} \mathrm{BW}$ as control and $0.01 \% \mathrm{w} / \mathrm{w}$ doses; and $\pm 6.5 \mathrm{~mL} / \mathrm{g} \mathrm{BW}$ as $0.02 \%$ dose. The amount of rats was determined by Federer's equation (n-1) $(t-1)>1$ (David and Arkeman, 2008), where " $t$ " is for number of treatment and "n" is for number of sample individuals. Approximately $250 \mathrm{~g}$ of fresh fecal samples were collected twice, before and after treatment (Day 0 and Day 28). The samples were then stored in $20{ }^{\circ} \mathrm{C}$. The abdominal circumference (AC), body length (BL), and body weight (BW) of all rats were measured on Day 0 and Day 28. By the end of study, euthanasia was performed using intraperitoneally Ketamine $80 \mathrm{mg} / \mathrm{kg}$ and Xylazine $10 \mathrm{mg} / \mathrm{kg}$, and intracardial Pentobarbital $200 \mathrm{mg} / \mathrm{kg}$. All procedures were approved by the ethical commission of Pusat Studi Satwa Primata, Bogor Agricultural University, Indonesia.

\section{Total Fecal Bacterial DNA Extraction}

Total fecal bacterial DNA was extracted using QIAamp ${ }^{\circledR}$ DNA Stool Minikit (Qiagen) with 15 minutes vortex with beads modification. Electrophoresis in $1.3 \%$ agarose gel was run using $5 \mu \mathrm{L}$ DNA at $60 \mathrm{~V}$ for 90 minutes in $1 \times$ TAE buffer, stained with ethidium bromide and visualized using UV light (Clement, 2014).

\section{Group-specific PCR Method}

The reaction was performed in total volume of $25 \mu \mathrm{L}$ consisted of $1 \mu \mathrm{L}$ DNA template, $12.5 \mu \mathrm{L}$ GoTaq Green ${ }^{\circledR}$ Master Mix (Promega), $1 \mu \mathrm{L}$ of each primer (Table 1) (Guo et al., 2008; Clement, 2014), and nuclease free water (NFW). PCR was performed using thermocycler machine with initial step at $94{ }^{\circ} \mathrm{C}$ for $5 \mathrm{~min}$; followed by 30 cycles of denaturation at $94{ }^{\circ} \mathrm{C}$ for $30 \mathrm{~s}$, annealing at $50{ }^{\circ} \mathrm{C}$ or $60{ }^{\circ} \mathrm{C}$ for 
Table 1. 16S rRNA Gene Targeted Group-specific Primers [23,28].

\begin{tabular}{lllll}
\hline Target Phyla & Primer & Sequence & $\begin{array}{l}\text { Size } \\
(\mathrm{bp})\end{array}$ & $\begin{array}{l}\text { Annealing } \\
\text { Temperature }\left({ }^{\circ} \mathrm{C}\right)\end{array}$ \\
\hline Bacteroidetes & Bact947f & GGARCATGTGGTTTAATTCGATGAT & 126 & 50 \\
& Bact1060r & AGCTGACGACAACCATGCAG & & \\
Firmicutes & Firm934f & GGAGYATGTGGTTTAATTCGAAGCA & 126 & 60 \\
& Firm1060r & AGCTGACGACAACCA & & \\
\hline
\end{tabular}

30s, and elongation at $72{ }^{\circ} \mathrm{C}$ for $1 \mathrm{~min}$; the final extension was at $72{ }^{\circ} \mathrm{C}$ for $20 \mathrm{~min}$ (Matsuki et al., 2004). Electrophoresis in $1.3 \%$ agarose gel was run using $2 \mu \mathrm{L}$ DNA product at $60 \mathrm{~V}$ for 90 minutes in $1 \times$ TAE buffer, stained with ethidium bromide and visualized using UV light.

\section{Gene Group-specific Cloning}

PCR products from the previous step were purified using QIA Quick PCR Purification Kit (Qiagen) and ligated into 3015 bp pGEM-T Easy vector (Promega) in a mix of $1 \mu \mathrm{L}$ pGEM-T Easy vector, $1 \mu \mathrm{L}$ T4 DNA Ligase, and $5 \mu \mathrm{L}$ Ligation Buffer 2x. PCR products were used as insert DNA in amount calculated by pGEM-T Easy suggestion using 6:1 (insert:vector) molar ratio. The ligation was performed in room temperature for 1 hour, and continued with overnight incubation in $4{ }^{\circ} \mathrm{C}$. Recombinant plasmid produced was further transformed to competent Escherichia coli $\mathrm{DH} 5 \alpha$ using $\mathrm{MgCl}_{2}$, $\mathrm{CaCl}_{2}$, and heat shock method. The transformants were plated on Luria Agar medium containing $100 \mu \mathrm{g} / \mathrm{mL}$ Ampicillin and $40 \mu \mathrm{g} / \mathrm{mL}$ X-Gal, incubated at $37{ }^{\circ} \mathrm{C}$ overnight. White colonies were picked and verified with PCR using M13 primers. The suspension of transformants that was used for medium plating was resuspended with Luria Broth containing $100 \mu \mathrm{g} / \mathrm{mL}$ Ampicillin and incubated for 12 hours at $37{ }^{\circ} \mathrm{C}$ with $120 \mathrm{rpm}$ shake. The plasmid of the culture was then isolated using Wizard ${ }^{\circledR}$ Plus SV Minipreps DNA Purification System (Promega).

\section{Standard Curve Construction for Amplification Efficiency Calculation}

A standard curve for each target bacteria phylum was constructed using tenfold dilutions, in duplicate, of the isolated recombinant plasmid with five points of concentrations (S1 and S2 Figs). The plasmid was diluted in NFW. The $i Q 5$ Multicolor Real-Time PCR Detection System (Bio-Rad) was used to retrieve cycle threshold $(\mathrm{Ct})$ value of each concentration using a mixture of $10 \mu \mathrm{L}$ KAPA SYBR Fast Green Master Mix (Bio-Rad), $1 \mu \mathrm{L}$ of each primer $(10 \mu \mathrm{M})$ (Table 1), $1 \mu \mathrm{L}$ plasmid, and NFW. The protocol used was $94{ }^{\circ} \mathrm{C}$ for $5 \mathrm{~min}$ at pre-denaturation stage continued with 40 cycles of $94{ }^{\circ} \mathrm{C}$ for $20 \mathrm{~s}, 50{ }^{\circ} \mathrm{C}$ or $60{ }^{\circ} \mathrm{C}$ for $20 \mathrm{~s}$ (Table 1) and $72{ }^{\circ} \mathrm{C}$ for $50 \mathrm{~s}$ (Matsuki et al., 2004; Guo et al., 2008).

The reaction efficiency (E) to calculate the ratio in relative quantification was retrieved using the equation as follows, using the slope from the standard curves (Bio-Rad, 2006).

$$
\mathrm{E}=10^{-1 / \text { slope }}
$$

\section{Fecal Bacteria DNA Relative Quantification}

RT-PCR amplification and detection were performed in the same reaction conditions used in standard curve construction, using $50 \mathrm{ng}$ total fecal bacteria DNA as the sample. The change ratio percentage of relative quantification result is calculated using the equation given in Bio-Rad RT-PCR Applications Guide (Bio-Rad, 2006).

Ratio $\left.{ }_{(\text {calibrator }}\right)=\mathrm{E}^{\mathrm{C}_{\mathrm{T}} \text { (calibrator)- } \mathrm{C}_{\mathrm{T}} \text { (test) }}$

Ratio $\frac{\text { Day zs }}{\left(\frac{1}{\text { Day } 0}\right)}=\mathrm{E}^{\mathrm{C}_{\mathrm{T}}\left(\text { Day 0)- } \mathrm{C}_{\mathrm{T}} \text { (Day 28) }\right.}$

Ratio $(\%)=$ Ratio $\times 100 \%$ 


\section{Statistical Analysis}

Statistical analysis was conducted using SPSS Statistic version 22.0 software. Independent samples t-test was performed to analyze the significant difference between before and after treatment rats' BMI. Mann-Whitney U test (two independent not normally distributed samples), and One-Way ANOVA test (independent distributed normally samples) were performed to analyze any significant differences in paired-samples for within each treatment group (comparing Day 0 to Day 28) and between each treatment groups of day (comparing all Day 0 and all Day 28) respectfully for the anthropometric data. For the bacterial ratio change data, Mann-Whitney U test was also used to analyze any significant differences in between treatment groups (Control and $0.01 \%$, also Control and $0.02 \%$ ), while Kruskal-Wallis test was used to analyze any significant differences between all treatment groups. The normality of the samples' distribution was tested using SapiroWilk test. Statistically significant difference was concluded using $\alpha=0.05$ and $\alpha=0.01$ where possible.

\section{Results and discussion}

\section{Obese-inducement}

After 14 days of $20 \%$ w/v sucrose supplementation to induce obesity, the BMI of the rats increased significantly $(\mathrm{p}<0.01)$ (Table 2). However, the BMI was decreased significantly after VCO supplementation in each treatment group compared to each control (Fig. 1). Sucrose supplementation was assumed to take role as an energy-dense supply of simple sugar, which could be metabolized and absorbed easily by rats' body metabolism as well as their gut microbiome that will be discussed later (Ley, 2010). Thus, it is logical that the termination of the supplementation led to the decrease of the rats' BMI due to decrease proportion of BL (Fig. 2) divided by squared AC (Fig. 3).

\section{Pro-obesity Effect on Anthropometric Parameters}

The anthropometric data collected in this study were body mass index (BMI), body weight (BW), and abdominal circumference (AC) as the parameters of obesity, and body length (BL) as additional data (Novelli et al. 2007). The results showed significant difference within group of all treatment for BMI $(p<0.01)$ with the trends of decreasing BMI along the period of treatment (Fig. 1). For the BW parameter, the trend of increasing BW within VCO-supplemented groups, both $0.01 \% \mathrm{w} / \mathrm{w}$ and $0.02 \% \mathrm{w} / \mathrm{w}$ were significant $(\mathrm{p}<0.01)$ (Fig. 4). There was also significant difference between treatment groups on Day 28 BW measurement ( $\mathrm{p}<0.05)$ (Fig. 4). Increasing trend was also appeared on $\mathrm{AC}$ parameter but only significantly different in $0.02 \%$ w/w VCO group (p<0.05) (Fig. 3).

Table 2. BMI change after Obesity-inducing Sucrose Supplementation

\begin{tabular}{lccc}
\hline Group & BW Mean & BL Mean & BMI Mean \\
\hline Day -14 & $107.86 \pm 6.18$ & $16.08 \pm$ & $0.42^{*} \pm 0.02$ \\
Day 0 & $167.40 \pm$ & $17.28 \pm$ & $0.56^{*} \pm 0.04$ \\
Day 28 & $176.31 \pm$ & $20.15 \pm$ & $0.46 \pm 0.41$ \\
\hline
\end{tabular}

*significantly different $(\mathrm{p}<0.01)$

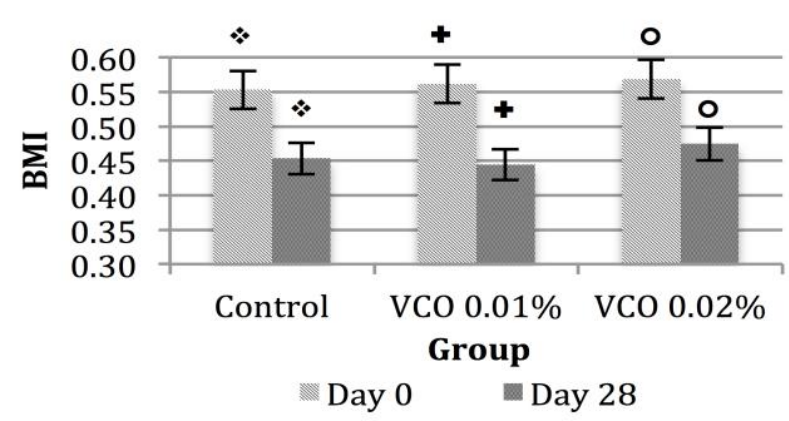

Figure 1. Rats' Body Mass Index (BMI) change before (Day 0) and after (Day 28) Treatment. $\square, \mathbf{4}, \square$ ) Significant difference within each treatment group (Mann-Whitney U test; $\mathrm{p}<0.01$ )

The increase of AC (Fig. 3) was found contrary with the clinical trial by Liau et al. (Liau et al., 2011) specifically and showed proobesity effect in general through the results in other anthropometrical parameters. Moreover, by the BW statistic test result it was shown that the more the VCO dose was, the higher the BW (Fig. 4). This might happen because of the dose of the VCO, which contained approximately 
$30 \%$ LCFAs of all fatty acids content (Nour et al., 2009). The doses used in this study was based on the mathematical calculation to convert the effective dose used in the research of Liau et al. (Liau et al., 2011) through the FDA's humanrat equal dose (Food and Drug Administration, 2005). The calculated dose might not be the optimum dose for VCO to stimulate its best antiobesity effect as the too high proportion of LCFA surmounted MCFA's anti-obesity effect. Different with MCFAs that were transported through portal vein directly to the liver for being used as the energy source thus increased the energy expenditure (Guturu and Duchini, 2012), LCFAs were transported by the lymph system from the intestine to subcutaneous tissue and could be used as building blocks of fat tissue hence increased the AC and other parameters.

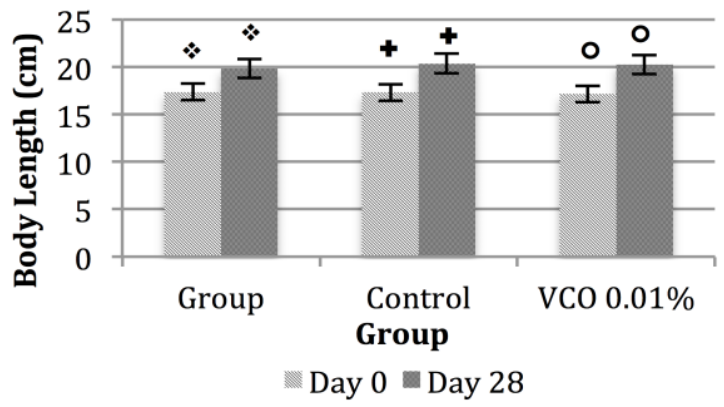

Figure 2. Rats' Body Length (BL) change before (Day 0) and after (Day 28) Treatment. $\square, \quad+, \square$ ) Significant difference within each treatment group (Mann-Whitney U test; $\mathrm{p}<0.01$ )

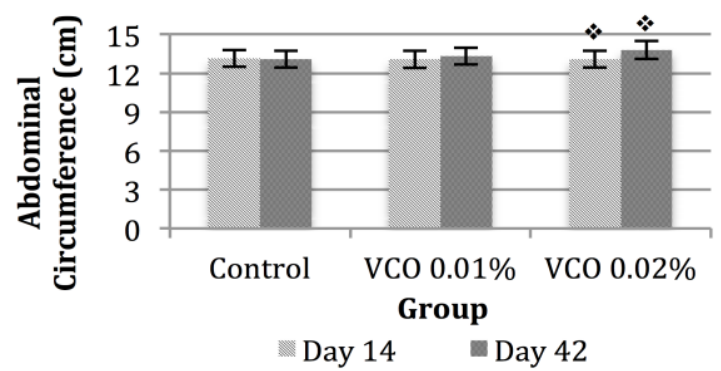

Figure 3. Rats' Abdominal Circumference (AC) change before (Day 0 ) and after (Day 28) Treatment. $\square$ ) Significant difference within $0.02 \% \mathrm{w} / \mathrm{w} \quad \mathrm{VCO}$ treatment groups (Mann-Whitney U test; $\mathrm{p}<0.05)$

\section{Pro-obesity Effect on Anthropometric Parameters}

The anthropometric data collected in this study were body mass index (BMI), body weight (BW), and abdominal circumference (AC) as the parameters of obesity, and body length (BL) as additional data (Novelli et al. 2007). The results showed significant difference within group of all treatment for BMI $(p<0.01)$ with the trends of decreasing BMI along the period of treatment (Fig. 1). For the BW parameter, the trend of increasing BW within VCO-supplemented groups, both $0.01 \% \mathrm{w} / \mathrm{w}$ and $0.02 \% \mathrm{w} / \mathrm{w}$ were significant $(\mathrm{p}<0.01)$ (Fig. 4). There was also significant difference between treatment groups on Day 28 BW measurement (p<0.05) (Fig. 4). Increasing trend was also appeared on $\mathrm{AC}$ parameter but only significantly different in $0.02 \%$ w/w VCO group (p<0.05) (Fig. 3).

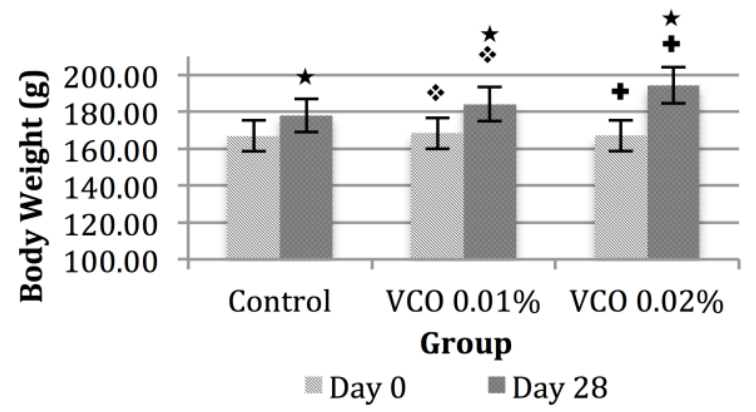

Figure 4. Rats' Body Weight (BW) change before (Day 0) and after (Day 28) Treatment. $\square, \quad \square+$ Significant difference within $0.01 \% \mathrm{w} / \mathrm{w} \mathrm{VCO}$ and $0.02 \% \mathrm{w} / \mathrm{w}$ VCO treatment groups (Mann-Whitney U test; $\mathrm{p}<0.01), \square \star$ ) Significant different between Day 28 groups (One-way ANOVA; $\mathrm{p}<0.05$ )

The increase of AC (Fig. 3) was found contrary with the clinical trial by Liau et al. (Liau et al., 2011) specifically and showed proobesity effect in general through the results in other anthropometrical parameters. Moreover, by the BW statistic test result it was shown that the more the VCO dose was, the higher the BW (Fig. 4). This might happen because of the dose of the VCO, which contained approximately 
$30 \%$ LCFAs of all fatty acids content (Nour et al., 2009). The doses used in this study was based on the mathematical calculation to convert the effective dose used in the research of Liau et al. (Liau et al., 2011) through the FDA's humanrat equal dose (Food and Drug Administration, 2005). The calculated dose might not be the optimum dose for VCO to stimulate its best antiobesity effect as the too high proportion of LCFA surmounted MCFA's anti-obesity effect. Different with MCFAs that were transported through portal vein directly to the liver for being used as the energy source thus increased the energy expenditure (Guturu and Duchini, 2012), LCFAs were transported by the lymph system from the intestine to subcutaneous tissue and could be used as building blocks of fat tissue hence increased the AC and other parameters.

\section{Anti-obesity Trend on Gut Microbiota Parameters}

VCO supplementation was previously assumed to likely promote changes in gut microbiome due to its reachability in various animal trials. In rats, the MCFA was found to reach the colonic lumen before entering the mesenteric blood, noted that the transport of MCFAs to the mesenteric blood exceeded 2-13 fold compared to SCFAs and LCFAs (Jørgensen et al., 2001). The MCFA contents in the portal vein blood was found only $26-27 \%$ recovery using octanoic acid (C8) and decanoic acid (C10) in pigs (Guillot et al., 1994). In other rat model, it was also showed that recovery of the MCFA in the lymphatic transport system was $7.3+/-0.9,26.3+/-2.4$, and $81.7+/-6.9 \%$ for caprylic acid (C8:0), capric acid (10:0), and lauric acid (12:0) (Mu and Høy, 2000). The remaining of the MCFAs that were undigested until reaching the colon were assumed to affect the environment of the colon, including the gut microbiota.

The Bacteroidetes and Firmicutes results both showed contrast relationship in higher VSO dose, regardless the non-significant statistical results (Figs 5 and 6, Table 3). Regardless the significance and relatively high in some standard deviations, roughly the contrast relationship was in accordance with the experiment conducted by
Turnbaugh et al. in regards of VCO as a fat in general (Turnbaugh et al., 2006). However, it doesn't seem contrary in terms of the hypothesis of this research, in which VCO is an exceptional fat source regarding its high content of medium chain fatty acids (MCFAs) that had been reported to promote anti-obesity effect to human and rats (Liau et al., 2011; Srivastava et al., 2013). Considering VCO's exceptionality, the result from this study could have many possibilities differ from the report from Turnbaugh et al. (Ley et al., 2006), including showing its anti-obesity effect by giving less stimulation to Firmicutes that previously reported linked with obesity.

Without any statistically significant result or trend, it was difficult to retrieve rigid interpretation in this study. However, insights about VCO's effect towards gut microbiota composition would still be retrieved from this study to initiate further examinations.

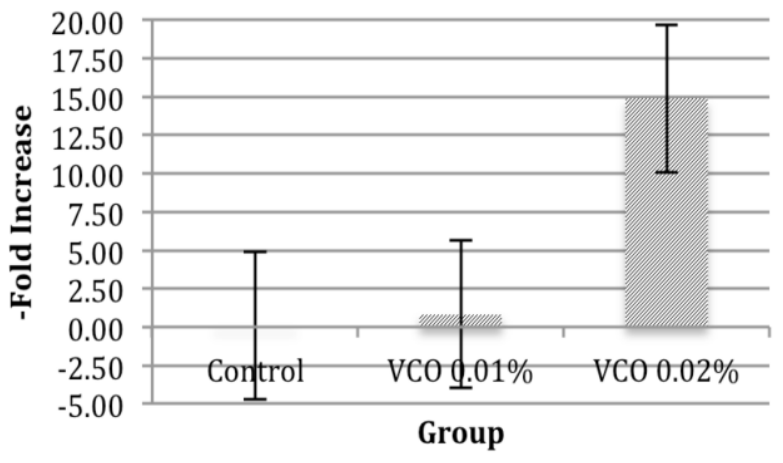

Figure 5. Bacteroidetes Ratio Change

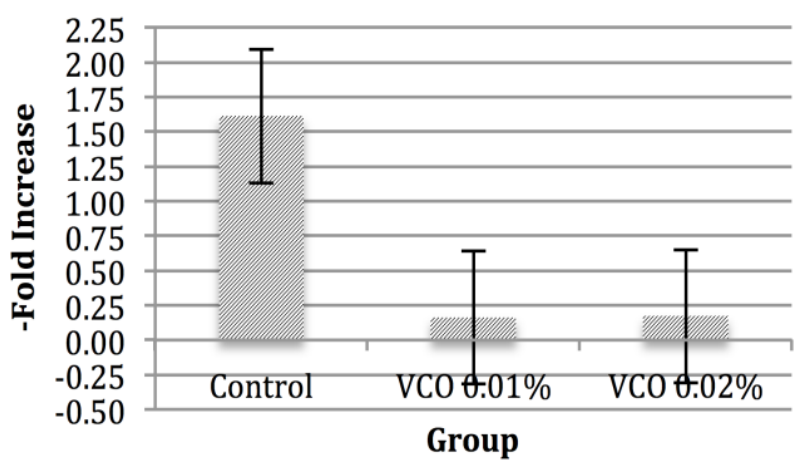

Figure 6. Firmicutes Ratio Change 
Table 3. Bacteroidetes and Firmicutes Ratio Change

\begin{tabular}{|l|l|l|l|l|}
\hline \multirow{2}{*}{ Group } & \multicolumn{4}{|c|}{ Average Change Ratio } \\
\cline { 2 - 5 } & \multicolumn{2}{|c|}{ Bacteroidetes } & \multicolumn{2}{c|}{ Firmicutes } \\
\cline { 2 - 5 } & Mean & Std. Dev. & Mean & Std. Dev. \\
\hline Control & 0.08 & 0.17 & 1.61 & 2.58 \\
\hline VCO 0.01\% & 0.84 & 1.76 & 0.16 & 0.12 \\
\hline VCO 0.02\% & 14.89 & 38.36 & 0.17 & 0.29 \\
\hline
\end{tabular}

Both bacteria phyla were chosen due to its domination in gut microbiota. Both phyla were mostly consisted of anaerobic bacteria, which dominated human and rats digestive system for more than 95\% (Turnbaugh et al., 2009). The proportion of both phyla in $\mathrm{F}: \mathrm{B}$ ratio was found to be correlated with the susceptibility of weight gain which further might lead to obesity (Ley et al., 2006).

The increase in Bacteroidetes might be caused by the presence of $\mathrm{VCO}$ as a new preferable source of energy in the digestive system that stimulated its growth. However, it was still unknown whether the MCFA could be readily digested by Bacteroidetes or else had to be metabolized first by themselves or by other microbes to produce SCFA as the main energy source of gut microbiota, which further examination was needed to discover the cellular mechanisms and interactions (Ley, 2010). Other possibility was that VCO suppressed other bacteria or microorganisms that played a role as Bacteroidetes' competitors, hence allowed Bacteroidetes to have higher growth rate as the VCO dose increases. However, to mention particular division(s) of which bacteria considered as the Bacteroidetes' competitors was still difficult due to the broad range of bacteria classes within the Bacteroidetes phylum itself (Krieg et al., 2011).

The Firmicutes result showed decreased ratio in VCO-supplemented groups. This could be caused by two possibilities. The first one is due to the stopped sucrose supplementation, as sucrose was a simple sugar that could easily absorbed by the bacteria (Ley, 2010). The second one is due to any possible flushing mechanism by the VCO physically. However, the second assumption was still difficult to meet any reports, moreover the Firmicutes phylum consisted of broad characteristics of bacteria classes, including their variable morphological characteristics that might affect the resistance against physical flush (Vos et al., 2011).

If with such limitations the Bacteroidetes and Firmicutes results in this study are still regarded as contrast, VCO would be regarded as an exceptional fat. The number of Firmicutes was found higher in obese individuals compared to lean individuals, while the number of Bacteroidetes showed the opposite. Beside that, germ-free mice that was resistant to diet-induced obesity lost its resistance after being transplanted the gut microbiota of obese donor, which had significantly higher relative abundance of Firmicutes $(p<0.05)$ compared to the lean donor. The germ-free mice that was transplanted with 'obese microbiota' was found to have greater increase of body fat gain compared to those that were transplanted with the 'lean microbiota'. Through continuous high-fat diet, the number of Firmicutes increased while Bacteroidetes decreased in mice (Turnbaugh et al., 2009).

The VCO supplementation in this study showed the opposite effect towards both phyla. In regards with the previous assumed explanations, VCO could be an example of how each fatty acids type would affect gut microbiota differently, giving emphasize on the importance of fat specification for further trials.

\section{Contrast Between Anthropometrical Data and Gut Microbiome Data}

By seeing the increase in Bacteroidetes and decrease in Firmicutes in this study as an against-obesity tendency as in the research of (Turnbaugh et al., 2009), the relationship compared to the anthropometrical results was be interesting. The VCO doses used was likely away from the optimum dose to promote the anti-obesity effect, while VCO loss in digestive tracts (Jørgensen et al., 2001; Mu and Høy, 2000) before the gut caused the gut microbiota to receive effective VCO dose. Regardless the 
doses used, the possible explanation was that the VCO had the characteristics that were unlike common fats for the gut microbiota, but still played the role as calorie source for the digestive tracts before the gut.

\section{Current Limitations and Further Examinations}

In this preliminary study, the rats' original gut microbiota level turned to play an important role to the data deviations resulted. The pre- and post-supplementation data were highly variable (S1 and S2 Tables), in which basal microbiota level was an important factor. It is highly recommended to conduct a conditioning of basal microbiota level of samples in order to achieve more statistically interpretable data.

In term of VCO-making process too, all the results retrieved in this experiment might be different when using VCO that was extracted with different methods. The chilling-thawing method was shown to be one of the best method to preserve the overall content of MCFA including its $\alpha$-tocopherol compared to fermentation, fresh-dry, and enzyme method (Mansor et al., 2012). Chilling method was assumed to damage the VCO contents less than heating method thus was suggested for the following studies.

To add the examination of SCFA in addition of this study's parameters is also highly recommended. Higher proportion of certain bacteria might stimulate the increase of nutrient metabolism in term of nutrient breakdown outside the bacteria cells. The broken-down nutrients then were made available to be absorbed by the epithelial cells of the host, for example by the fermentation of the dietary fiber to produce SCFA that could be utilized by the host as the source of energy. More than that, the produced SCFAs could bind to GPR41, a G protein coupled receptor that binds SCFA, thus increased the circulating level of Peptide YY (PYY). PYY was an enteroendocrine hormone that reduced gut motility hence increased the absorption of SCFA (Thakur et al., 2014).

Different SCFA stimulated different metabolic responses. Acetate, the principal
SCFA in the colon, had been shown to increase cholesterol synthesis after absorption. In contrast, propionate that stimulated liver gluconeogenesis and lipogenesis had been shown to decrease cholesterol synthesis. Butyrate has been shown nourishing colonic mucosa that promoted cell differentiation, cell cycle arrest, and apoptosis of transformed colonocytes thus could prevent cancer (Wong et al., 2006). Given that, metabolic change due to the increase of SCFA was likely dependent on which SCFA to be produced. In case of obesity, it was likely related mostly with propionate that promoted lipogenesis and gluconeogenesis. SCFA itself had been used as another parameter beside the $\mathrm{F}: \mathrm{B}$ ratio to examine obesity. It was shown that the significant decrease of $\mathrm{F}: \mathrm{B}$ ratio between overweight $(p<0.01)$ and obese $(p<0.05)$ compared to lean volunteers went along with the significant increase in SCFA level towards in same group patterns $(p<0.05)$ (Schwiertz et al., 2010). Further examination was needed whether it was possible that the MCFA was degraded into SCFAs and also which SCFA was produced. The explanation would be useful to give a clue how MCFA metabolism caused its anti-obesity effect in gut environment.

\section{Conclusion}

This research showed a pro-obesity effect in anthropometrical parameters of BW, BL, and AC after VCO supplementation. On the other hand, with the limitation of no significant data and some high standard deviations, the $0.02 \%$ VCO supplementation promoted tendencies on the rough ratio changes of Bacteroidetes and Firmicutes to increase and decrease respectfully, which went towards the against-obesity effect. Further examinations using samples with normalized basal gut microbiota, VCO's optimum concentrations to promote its clinical anti-obesity effect, cold-processing VCOmaking method, and SCFA as additional parameters are needed.

\section{Acknowledgements}

Special thanks for PT Indofood CBP Sukses Makmur's Indofood Riset Nugraha 2014/2015, Dr. Susan Soka, M.Sc., Frans Ahnan, 
PT EMBRIO Biotekindo, Prof. Dr. F. G. Winarno, and Adi Yulandi.

\section{References}

Asia Pacific Coconut Community (2015) APCC Standard for Virgin Coconut Oil. [online]. Available from: http://www.apccsec.org/ document/VCO-STANDARDS.pdf (Accessed 22 July 2015). [online]. Available from: http://www.apccsec.org/ document/VCO-STANDARDS.pdf (Accessed 22 July 2015).

Bäckhed, F. et al. (2005) Host-bacterial mutualism in the human intestine. Science (New York, N.Y.). [Online] 307 (5717), 1915-1920.

Bio-Rad (2006) Real-Time Guide Applications Guide. [online]. Available from: http://www.bio-rad.com/webroot/web/ pdf/ lsr/literature/Bulletin_5279.pdf (Accessed 22 July 2015). [online]. Available from: http://www.bio-rad.com/webroot/web/pdf/ lsr/literature/Bulletin_5279.pdf (Accessed 22 July 2015).

Clement, S. (2014) Impact of Amorphophallus campanulatus supplementation on gut microbiota composition in DIO-Sprague Dawley Rat. Jakarta, Indonesia: Atma Jaya Catholic University of Indonesia.

David \& Arkeman, H. (2008) Evaluation of the oral toxicity of formaldehyde in rats. 27 (3), 106-112.

Dorak, M. T. (2007) Real-Time PCR. Garland Science.

Everard, A. et al. (2011) Responses of gut microbiota and glucose and lipid metabolism to prebiotics in genetic obese and diet-induced leptin-resistant mice. Diabetes. [Online] 60 (11), 2775-2786.

Food and Drug Administration (2005) Guidance for Industry: Estimating the Maximum Safe Starting Dose in Initial Clinical Trials for Therapeutics in Adult Healthy Volunteers. [online]. Available from: http://www.fda.gov/downloads/Drugs/Gui dances/UCM078932.pdf (Accessed 22
July 2015). [online]. Available from: http://www.fda.gov/downloads/Drugs/Gui dances/UCM078932.pdf (Accessed 22 July 2015).

Guillot, E. et al. (1994) Intestinal absorption of medium chain fatty acids: in vivo studies in pigs devoid of exocrine pancreatic secretion. The British Journal of Nutrition. 72 (4), 545-553.

Guo, X. et al. (2008) Development of a real-time PCR method for Firmicutes and Bacteroidetes in faeces and its application to quantify intestinal population of obese and lean pigs. Letters in Applied Microbiology. [Online] 47 (5), 367-373.

Guturu, P. \& Duchini, A. (2012) Etiopathogenesis of Nonalcoholic Steatohepatitis: Role of Obesity, Insulin Resistance and Mechanisms of Hepatotoxicity. International Journal of Hepatology. [Online] 2012e212865.

Herrera, B. M. et al. (2011) Genetics and epigenetics of obesity. Maturitas. [Online] 69 (1), 41-49.

Jørgensen, J. R. et al. (2001) In vivo absorption of medium-chain fatty acids by the rat colon exceeds that of short-chain fatty acids. Gastroenterology. [Online] 120 (5), 1152-1161.

Kalliomäki, M. et al. (2008) Early differences in fecal microbiota composition in children may predict overweight. The American Journal of Clinical Nutrition. 87 (3), 534538.

Krieg, N. R. et al. (2011) Bergey's Manual of Systematic Bacteriology: Volume 4: The Bacteroidetes, Spirochaetes, Tenericutes (Mollicutes), Acidobacteria, Fibrobacteres, Fusobacteria, Dictyoglomi, Gemmatimonadetes, Lentisphaerae, Verrucomicrobia, Chlamydiae, and Planctomycetes. Springer Science \& Business Media.

Lekanne Deprez, R. H. et al. (2002) Sensitivity and accuracy of quantitative real-time polymerase chain reaction using SYBR 
green I depends on cDNA synthesis conditions. Analytical Biochemistry. [Online] 307 (1), 63-69.

Ley, R. E. et al. (2006) Microbial ecology: human gut microbes associated with obesity. Nature. [Online] 444 (7122), 1022-1023.

Ley, R. E. (2010) Obesity and the human microbiome. Current Opinion in Gastroenterology. [Online] 26 (1), 5-11.

Liau, K. M. et al. (2011) An Open-Label Pilot Study to Assess the Efficacy and Safety of Virgin Coconut Oil in Reducing Visceral Adiposity. ISRN Pharmacology. [Online] 2011.

Mansor, T. S. T. et al. (2012) Physicochemical properties of virgin coconut oil extracted from different processing methods. International Food Research Journal. 19 (3), 837-845.

Mariat, D. et al. (2009) The Firmicutes/ Bacteroidetes ratio of the human microbiota changes with age. $B M C$ Microbiology. [Online] 9 (1), 123.

Matsuki, T. et al. (2004) Use of 16S rRNA genetargeted group-specific primers for realtime PCR analysis of predominant bacteria in human feces. Applied and Environmental Microbiology. [Online] 70 (12), 7220-7228.

Mu, H. \& Høy, C. E. (2000) Effects of different medium-chain fatty acids on intestinal absorption of structured triacylglycerols. Lipids. 35 (1), 83-89.

National Human Genome Research Institute (2015) Metagenomics. Talking Glossary of Genetic Terms [online]. Available from: http://www.genome.gov/glossary/index.cf $\mathrm{m} ? \mathrm{id}=503$ (Accessed 22 July 2015). [online]. Available from: http://www.genome.gov/glossary/index.cf m?id=503 (Accessed 22 July 2015).

Nevin, K. G. \& Rajamohan, T. (2006) Virgin coconut oil supplemented diet increases the antioxidant status in rats. Food Chemistry. [Online] 99 (2), 260-266.

Nour, A. H. et al. (2009) Demulsification of virgin coconut oil by centrifugation method: a feasibility study. International Journal of Chemical Technology. [Online] 1 (2), 59-64.

Novelli, E. L. B. et al. (2007) Anthropometrical parameters and markers of obesity in rats. Laboratory Animals. [Online] 41 (1), 111119.

Schwiertz, A. et al. (2010) Microbiota and SCFA in lean and overweight healthy subjects. Obesity (Silver Spring, Md.). [Online] 18 (1), 190-195.

Srivastava, Y. et al. (2013) Hypocholesterimic effects of cold and hot extracted virgin coconut oil (VCO) in comparison to commercial coconut oil: Evidence from a male wistar albino rat model. Food Science and Biotechnology. [Online] 22 (6), 1501-1508.

Thakur, A. K. et al. (2014) Gut-Microbiota and Mental Health: Current and Future Perspectives. J Pharmacol Clin Toxicol. 21016.

Tlaskalová-Hogenová, H. et al. (2011) The role of gut microbiota (commensal bacteria) and the mucosal barrier in the pathogenesis of inflammatory and autoimmune diseases and cancer: contribution of germ-free and gnotobiotic animal models of human diseases. Cellular \& Molecular Immunology. [Online] 8 (2), 110-120.

Turnbaugh, P. J. et al. (2009) A core gut microbiome in obese and lean twins. Nature. [Online] 457 (7228), 480-484.

Turnbaugh, P. J. et al. (2006) An obesityassociated gut microbiome with increased capacity for energy harvest. Nature. [Online] 444 (7122), 1027-1131.

Vos, P. et al. (2011) Bergey's Manual of Systematic Bacteriology: Volume 3: The 
Cord 2016, 32 (1)

Firmicutes. Springer Science \& Business Media.

Wong, J. M. W. et al. (2006) Colonic health: fermentation and short chain fatty acids. Journal of Clinical Gastroenterology. 40 (3), 235-243.

World Health Organization (2015) Obesity and overweight [online]. Available from: http://www.who.int/mediacentre/factsheets /fs311/en/ (Accessed 22 July 2015).

Zimmermann, R. et al. (2004) Fat mobilization in adipose tissue is promoted by adipose triglyceride lipase. Science (New York, N.Y.). [Online] 306 (5700), 1383-1386. 